${ }^{1}$ Universidade do Estado do Amazonas. Av. Djalma Batista 2470, Chapada. 69050-010 Manaus AM Brasil.valdirsoares.vs@ gmail.com

${ }^{2}$ Instituto de Física, Universidade de São Paulo. São Paulo SP Brasil. ${ }^{3}$ Departamento de Endemias Samuel Pessoa, Escola Nacional de Saúde Pública, Fiocruz. Rio de Janeiro RJ Brasil.

\title{
Distribuição espacial de queimadas e mortalidade em idosos em região da Amazônia Brasileira, 2001 - 2012
}

\author{
Spatial distribution of biomass burning and mortality \\ among the elderly in a Brazilian Amazon region, 2001 - 2012
}

Abstract The burning of biomass has a significant impact on the Amazon ecosystem in the dry season due to the emissions of air pollutants. The effects on the health of the population, especially in the region of the arc of deforestation, has been the subject of recent studies. The scope of this study was to evaluate the spatial distribution of biomass burning and mortality from respiratory and cardiovascular diseases among the elderly in the state of Rondonia in the period from 2001 to 2012. Mortality data were obtained through the Mortality Information System of the Ministry of Health. Biomass burning data were provided by the National Institute for Space Research. The Kernel estimator was used. The highest mortality rates were observed in the central-east and southeast of Rondônia. The focuses of the fires were concentrated in the northern part of the state, though with a significant amount in other regions. The spatial distribution of the hot areas of mortality and fires were not directly associated. However, fires were observed in all municipalities in the state. Pollutants emitted from biomass burning can be transported thousands of kilometers from the source areas and influence the health of the elderly.

Key words Spatial distribution, Biomass burning, Elderly, Mortality, Amazon
Resumo As queimadas exercem relevante impacto sobre o ecossistema amazônico na estação seca com as emissões de poluentes atmosféricos. Os efeitos sobre a saúde das populações, principalmente na região do arco do desmatamento, tem sido objeto de recentes estudos. O presente estudo teve como objetivo avaliar a distribuição espacial dos focos de queimadas e da mortalidade por doenças respiratórias e cardiovasculares em idosos, no Estado de Rondônia, no período de 2001 a 2012. Os dados de mortalidade foram obtidos por meio do Sistema de Informação de Mortalidade, do Ministério da Saúde. Dados de focos de queimadas foram disponibilizados pelo Instituto Nacional de Pesquisas Espaciais. Utilizou-se o estimador de Kernel. As maiores taxas de mortalidade foram observadas no centro-leste e sudeste de Rondônia. Os focos de queimadas concentraram-se na porção norte do estado, mas com quantidade relevante em outras regiões. As distribuições espaciais das áreas quentes de taxa de mortalidade e de queimadas não se mostraram diretamente associadas. Entretanto, as queimadas foram observadas em todos os municípios do estado. Os poluentes emitidos na queima podem ser transportados por milhares de quilômetros das áreas de origem e influenciar a saúde de idosos.

Palavras-chave Distribuição espacial, Queimadas, Idosos, Mortalidade, Amazônia 


\section{Introdução}

A queima de biomassa está entre os principais contribuintes mundiais para a emissão de poluentes atmosféricos, incluindo os gases de efeito estufa e material particulado, resultando, em muitos casos, a exposição humana a elevados níveis de poluentes do ar. Incêndios florestais e queima de biomassa deliberada, embora fontes intermitentes de poluição do ar, representam grandes fontes de poluição por combustão globalmente ${ }^{1}$.

Segundo a Organização Mundial de Saúde², a exposição às partículas em suspensão tem sido associada a uma série de resultados adversos para a saúde, incluindo alterações transitórias às vias respiratórias, o comprometimento da função pulmonar, o aumento do risco de morte por doenças cardiovasculares e respiratórias. No mundo, o material particulado é estimado em causar cerca de $8 \%$ das mortes por câncer de pulmão, $5 \%$ por doença cardiopulmonar e cerca de $3 \%$ por infecções respiratórias ${ }^{2}$.

Johnston et al. ${ }^{3}$, em um estudo do período de 1997 a 2006, estimaram a mortalidade média global atribuível às queimadas em cerca de 339 mil mortes por ano, onde as regiões mais atingidas foram observadas na África subsaariana, no Sudeste Asiático e em porções da América do Sul.

De acordo com a Organização Meteorológica Mundial ${ }^{4}$, os centros urbanos da América do Sul não só estão sujeitos à poluição do ar que se origina nas próprias cidades, pela queima de combustíveis fósseis e atividades industriais, mas também são notavelmente afetados pela queima de biomassa em larga escala. Apesar das reivindicações internacionais e do compromisso do governo brasileiro de limitar o desmatamento e as queimadas na Amazônia, a ineficiência das ações atuais torna aberta a discussão da problemática dentro das próximas décadas.

Estima-se que cerca de $80 \%$ da queima de biomassa ocorre nos trópicos, resultado da crescente pressão demográfica nestas áreas onde o fogo é utilizado extensivamente como uma prática de manejo do solo. Apesar de anos de estudos científicos sobre os impactos dos poluentes atmosféricos em áreas urbanas e a atenção da mídia em relação ao desmatamento e aos incêndios florestais, acidentais ou intencionais, os potenciais efeitos a saúde das populações têm sido pouco estudados pela comunidade científica ${ }^{5}$. Pesquisas anteriores já destacaram os efeitos dos poluentes originados na queima de biomassa sobre a morbimortalidade dos grupos biologicamente vulneráveis, as crianças e os idosos ${ }^{6,7}$.
Entretanto, apesar da literatura disponível sobre a relação saúde e poluentes atmosféricos em centros urbanos, ainda são poucos os estudos que abordam os efeitos à saúde das populações expostas à fumaça das queimadas, principalmente na região Amazônica ${ }^{8}$. Pesquisas relacionadas às queimadas devem considerar a influência de diversos fatores que poderiam ter um ou mais efeitos diretos e indiretos, com consequência aos ecossistemas. São inúmeras as variáveis que podem influenciar a ocorrência de agravos à saúde e separá-las para determinar o efeito isolado é bastante difícil, recomendando a existência de metodologias específicas para cada caso à exposição aos poluentes?.

A composição química da atmosfera amazônica sofre grandes mudanças na época da seca, devido às emissões de gases traço e partículas de aerossóis provenientes de queimadas de pastagens e florestas, os quais exercem efeitos diretos e indiretos no clima e funcionamento do ecossistema amazônico ${ }^{10}$. A saúde das populações tem sido afetada de forma significativa, principalmente na região do arco do desmatamento ${ }^{11}$.

O Estado de Rondônia apresentou relevante crescimento populacional, principalmente a partir da década de 1960, promovido por políticas do governo de desenvolvimento socioeconômico e ocupação da Amazônia brasileira, que incentivaram o fluxo migratório à região e o crescimento de atividades econômicas relacionadas ao uso do solo, como exploração de madeira, agricultura e pecuária ${ }^{12}$. O incremento de atividades de desmatamento e queimadas é parte consequente deste cenário.

O estudo teve como objetivo avaliar a distribuição espacial dos focos de queimadas e da mortalidade por doenças respiratórias e cardiovasculares em idosos, no Estado de Rondônia, no período de 2001 a 2012.

\section{Métodos}

Trata-se de um estudo ecológico da distribuição espacial das taxas de mortalidade por doenças respiratórias e cardiovasculares em maiores de 60 anos de idade e de focos de queimadas nos municípios do Estado de Rondônia, no período de $1^{\circ}$ de janeiro de 2001 a 31 de dezembro de 2012.

Rondônia está situado na porção sul da região Amazônica brasileira e sua área é de aproximadamente 238 mil km² (Figura 1). Em 2014, apresentou uma população estimada em 1.748.531 habitantes. De acordo com o último censo, em 2010, 


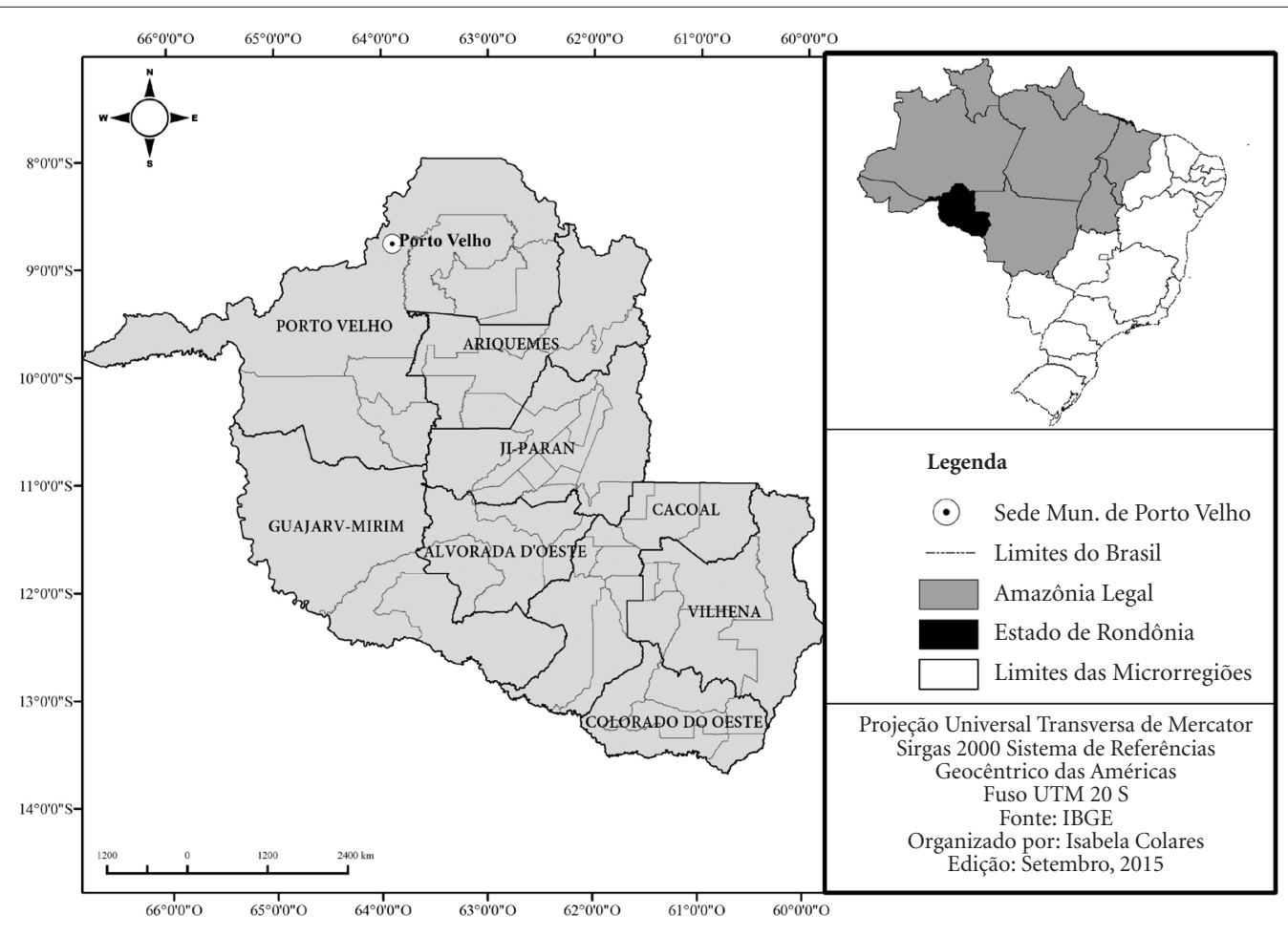

Figura 1. Localização do Estado de Rondônia, Amazônia brasileira.

a população acima de 60 anos foi registrada em 112.285 habitantes, $7 \%$ da população do estado. Possui 52 municípios, distribuídos em oito microrregiões: Alvorada D'Oeste, Ariquemes, Cacoal, Colorado D'Oeste, Guajará-Mirim, Ji-Paraná, Porto Velho e Vilhena.

Rondônia integra uma região conhecida como 'Arco do Desmatamento', cujos limites abrangem desde o sudeste do Maranhão, incluindo o norte do Tocantins, o sul do Pará, o norte do Mato Grosso, o estado de Rondônia, o sul do Amazonas, até o sudeste do Acre. As queimadas ocorrentes na região são quase sempre associadas ao desmatamento, pois é quando, após o desmate, agricultores e fazendeiros queimam suas terras para converter as florestas em áreas de lavouras ou pastagens ${ }^{13}$. Em 2014, o Arco do Desmatamento concentrou aproximadamente $67 \%$ das queimadas que ocorreram no Brasil. Neste mesmo ano, entre os estados da Amazônia, Rondônia apresentou 5\% dos focos estimados ${ }^{14}$.

Os dados de mortalidade foram obtidos por meio do Sistema de Informação de Mortalidade $(\mathrm{SIM})^{15}$, do Ministério da Saúde. As causas de mortalidade por doenças respiratórias e cardiovasculares (Capítulo X, J00-J99; e Capítulo IX, I00-I99, respectivamente) foram codificadas de acordo com a Décima Revisão da Classificação
Estatística Internacional de Doenças e Problemas Relacionados à Saúde (CID-10). As informações demográficas e as bases cartográficas utilizadas no estudo foram disponibilizadas pelo Instituto Brasileiro de Geografia e Estatística (IBGE) ${ }^{16}$. A série temporal dos focos de queimadas foi obtida no banco de dados do Instituto Nacional de Pesquisas Espaciais (INPE) ${ }^{14}$. Foram utilizados os considerados "satélites de referência", NOAA 12 (até 9 de agosto de 2007) e AQUA_M-T (de 9 de agosto de 2007 até a presente data), os quais permitem analisar as tendências espaciais e temporais dos focos, ao longo dos anos, em uma região.

A taxa de mortalidade foi calculada mediante a divisão do número de óbitos por doenças respiratórias e do aparelho circulatório em adultos, com idade acima de 60 anos, pela população total dessa idade, por município, no respectivo ano, multiplicado por mil. As taxas foram padronizadas de forma direta, pela população de idosos de Rondônia, para permitir a comparação entre os 52 municípios do Estado. Para tanto, foi necessário estratificar o grupo de maiores de sessenta anos em três subgrupos: de 60 a 69, 70 a 79 e acima de 80 anos.

A distribuição espacial foi estudada a partir da construção de mapas resultantes da estimativa de densidade de Kernel (estimador de Kernel), para 
a identificação das áreas de maiores concentrações dos desfechos e relevância ("áreas quentes”) dos indicadores de análise. As “áreas quentes” são uma aproximação das possíveis áreas geográficas de risco, uma vez que representam a concentração de casos ou focos no espaço. Essas análises foram conduzidas pelo programa TerraView 3.2.0, com os seguintes parâmetros: grade com um número de colunas em 600 sobre a região, algoritmo de função quártica e raio adaptativo. No cálculo matricial foram levados em consideração os centroides dos municípios. O nível de significância de $5 \%$ foi considerado em todas as análises. $\mathrm{O}$ presente trabalho é parte de um projeto maior, "Avaliação de Impacto à Saúde. A construção de uma ferramenta para a gestão socioambiental de projetos de desenvolvimento na Amazônia", e foi aprovado pelo Comitê de Ética em Pesquisa da Escola Nacional de Saúde Pública.

\section{Resultados}

Entre $1^{\circ}$ de janeiro de 2001 e 31 de dezembro de 2012 foram registrados 112.863 focos de queimadas em Rondônia, estimados pelos satélites de referência, NOAA e AQUA. A média anual de queimadas no estado foi de 9.405 focos. O município de maior incidência de queimadas foi Porto Velho, o qual obteve $17,4 \%$ dos registros totais da série temporal, seguidos de Nova Mamoré $(6,1 \%)$, Costa Marques (5,2\%) e São Francisco do Guaporé (5\%) (Tabela 1). Neste período, 2005 foi o ano de maior número de focos registrados, no estado de Rondônia, com um somatório de 17.818 focos estimados.

No período de 2001 a 2012, as taxas de mortalidade padronizadas por doenças cardiovasculares e respiratórias apresentaram, respectivamente, média anual de 10,0 e 3,1 óbitos em idosos por mil idosos, em Rondônia.

Para doenças cardiovasculares, o município de Ouro Preto do Oeste, situado à porção centro -leste do estado, apresentou a maior média anual de taxa de mortalidade, 14,1/1.000 idosos (Tabela 1). Ao longo da série temporal, a maior taxa anual foi observada em 2008, no município de Pimenteiras do Oeste, no sul de Rondônia, com $39,1 / 1.000$ idosos. No período de estudo, foram registrados 12.696 óbitos em idosos por doenças do aparelho circulatório (60-69, 30\%; 70-79, $38 \%$; acima de 80 anos, $32 \%$ ), sendo o desfecho de maior prevalência entre todas as causas de óbito observadas nesta faixa etária entre 2001 a 2012, com 35\% do total. Acidente vascular ce- rebral (I64) e Infarto agudo do miocárdio (I21) foram os principais motivos de óbito observados, respectivamente, com 22 e $21 \%$ dos diagnósticos.

Em relação às doenças do aparelho respiratório, foi observada no município de Ji-Paraná, situado na porção leste de Rondônia, a maior média anual de taxa de mortalidade, 6,0/1.000 idosos (Tabela 1). A maior taxa anual foi registrada em 2005, no município de Ministro Andreazza, no leste do estado, com 14,9/1.000 idosos. Ao longo da série avaliada foram registrados 4.427 óbitos em idosos por doenças respiratórias (60-69, 25\%; $70-79,37 \%$; acima de 80 anos, 39\%), que representaram a terceira causa mais frequente entre a mortalidade nesta faixa etária, com cerca de 12\% do total. Doença Pulmonar Obstrutivo Crônica (J44) e Pneumonia (J18) foram as causas de óbito mais frequentes, respectivamente, com cerca de 41 e $25 \%$ dos registros.

A distribuição espacial dos óbitos por doenças cardiovasculares e respiratórias em idosos apresentou configuração espacial semelhante em toda a série (Figuras 2 e 3 ). As maiores taxas de mortalidade para os desfechos foram observadas, em geral, dentro das microrregiões de Ji-Paraná, Alvorada do Oeste e Cacoal, situadas no centro-leste e sudeste de Rondônia, em municípios como Ouro Preto do Oeste, Ji-Paraná, Rolim de Moura, São Francisco do Guaporé, Alta Floresta do Oeste e Cacoal. O município de Porto Velho, capital do estado, situada na porção norte, apresentou uma das maiores médias anuais de taxa de mortalidade em doenças cardiovasculares (13,6/1000 idosos) e respiratórias (5,8/1000 idosos).

Ao longo da série, os focos de queimadas foram observados em maior número na região norte do estado, com destaque para os municípios da microrregião de Porto Velho, como Porto Velho, Nova Mamoré, Buritis e Machadinho do Oeste, onde as “áreas quentes" destacaram-se de forma concentrada (Figura 4). Nos anos de 2001, 2002, 2003, 2004, 2005, 2007 e 2010 as concentrações de queimadas mostraram-se elevadas em diversas microrregiões de Rondônia.

\section{Discussão}

Este é o primeiro estudo que avalia a distribuição espacial da mortalidade em idosos por doenças circulatórias e respiratórias e das queimadas, no estado de Rondônia. A distribuição espacial das "áreas quentes" das taxas de mortalidade por doenças cardiovasculares e respiratórias em idosos e das queimadas, no estado de Rondônia, no perío- 
Tabela 1. Estatística descritiva das taxas padronizadas de mortalidade por doenças circulatórias e respiratórias em idosos e de focos de calor, nos municípios do estado de Rondônia, no período de 2001 a 2012.

\begin{tabular}{|c|c|c|c|c|c|c|c|c|c|}
\hline \multirow[b]{2}{*}{ Município } & \multicolumn{3}{|c|}{ Doenças circulatórias } & \multicolumn{3}{|c|}{ Doenças respiratórias } & \multicolumn{3}{|c|}{ Focos de calor } \\
\hline & $\operatorname{Max}$ & Min & $\begin{array}{l}\text { Média } \\
\text { anual }\end{array}$ & Max & Min & $\begin{array}{l}\text { Média } \\
\text { anual }\end{array}$ & $\operatorname{Max}$ & Min & $\begin{array}{c}\text { Somatório da } \\
\text { série }\end{array}$ \\
\hline Alta Floresta D’Oeste & 18,2 & 6,3 & 12,8 & 6,9 & 1,5 & 4,2 & 482 & 0 & 2161 \\
\hline Alto Alegre dos Parecis & 12,3 & 2,9 & 7,4 & 10,5 & 0,0 & 3,3 & 341 & 26 & 1771 \\
\hline Alto Paraíso & 14,9 & 3,0 & 8,8 & 7,0 & 0,0 & 2,8 & 481 & 59 & 2331 \\
\hline Alvorada D’Oeste & 23,8 & 6,3 & 10,9 & 5,5 & 1,1 & 2,9 & 167 & 0 & 928 \\
\hline Ariquemes & 17,5 & 7,3 & 12,5 & 7,5 & 1,5 & 4,1 & 525 & 52 & 2647 \\
\hline Buritis & 26,4 & 4,8 & 14,0 & 11,5 & 0,0 & 3,6 & 867 & 141 & 5235 \\
\hline Cabixi & 20,7 & 3,0 & 9,4 & 11,4 & 1,4 & 5,7 & 187 & 7 & 688 \\
\hline Cacaulândia & 19,2 & 3,5 & 12,2 & 5,0 & 0,0 & 1,0 & 248 & 7 & 1163 \\
\hline Cacoal & 18,4 & 8,9 & 12,9 & 7,0 & 2,6 & 4,2 & 297 & 18 & 1052 \\
\hline Campo Novo de Rondônia & 10,8 & 0,0 & 6,6 & 10,9 & 0,0 & 2,8 & 522 & 63 & 3151 \\
\hline Candeias do Jamari & 14,3 & 1,8 & 8,0 & 7,5 & 0,0 & 3,9 & 580 & 91 & 4211 \\
\hline Castanheiras & 16,7 & 0,0 & 6,3 & 8,1 & 0,0 & 2,3 & 81 & 5 & 364 \\
\hline Cerejeiras & 16,3 & 8,6 & 13,4 & 7,1 & 2,4 & 4,3 & 206 & 18 & 1042 \\
\hline Chupinguaia & 15,3 & 0,0 & 4,7 & 9,2 & 0,0 & 2,9 & 722 & 85 & 3688 \\
\hline Colorado do oeste & 13,6 & 9,2 & 11,5 & 6,1 & 1,3 & 4,0 & 90 & 1 & 269 \\
\hline Corumbiara & 11,9 & 3,1 & 6,6 & 9,8 & 0,0 & 2,6 & 231 & 23 & 1404 \\
\hline Costa Marques & 20,5 & 6,0 & 13,5 & 7,4 & 0,0 & 3,8 & 1286 & 103 & 5862 \\
\hline Cujubim & 17,5 & 0,0 & 9,8 & 6,1 & 0,0 & 3,1 & 639 & 113 & 4235 \\
\hline Espigão D’Oeste & 19,6 & 3,3 & 11,2 & 5,7 & 1,3 & 2,9 & 343 & 0 & 1263 \\
\hline Governador Jorge Teixeira & 15,0 & 0,0 & 5,4 & 2,3 & 0,0 & 0,8 & 220 & 9 & 1161 \\
\hline Guajará-Mirim & 13,6 & 7,4 & 10,7 & 5,9 & 0,9 & 3,4 & 397 & 0 & 1609 \\
\hline Itapuã do Oeste & 11,9 & 0,0 & 5,2 & 10,9 & 0,0 & 4,2 & 266 & 48 & 1466 \\
\hline Jaru & 13,9 & 4,9 & 8,5 & 5,9 & 1,3 & 2,6 & 180 & 4 & 891 \\
\hline Ji-Paraná & 20,0 & 9,7 & 13,9 & 9,8 & 2,8 & 6,0 & 207 & 17 & 921 \\
\hline Machadinho D'Oeste & 12,8 & 3,2 & 8,3 & 3,6 & 0,7 & 2,0 & 1030 & 0 & 5202 \\
\hline Ministro Andreazza & 15,2 & 2,9 & 9,3 & 14,9 & 0,0 & 3,2 & 107 & 1 & 298 \\
\hline Mirante da serra & 23,7 & 5,1 & 13,3 & 6,1 & 0,0 & 2,4 & 69 & 1 & 383 \\
\hline Monte Negro & 22,4 & 5,1 & 11,3 & 6,3 & 0,0 & 2,5 & 270 & 30 & 1372 \\
\hline Nova Brasilândia D’Oeste & 20,6 & 6,7 & 13,3 & 11,6 & 1,3 & 4,0 & 77 & 0 & 509 \\
\hline Nova Mamoré & 21,4 & 8,3 & 11,1 & 6,3 & 0,0 & 2,8 & 1223 & 188 & 6871 \\
\hline Nova União & 14,3 & 2,1 & 8,9 & 4,3 & 0,0 & 2,3 & 58 & 5 & 308 \\
\hline Novo Horizonte do Oeste & 21,6 & 3,5 & 9,5 & 7,1 & 0,0 & 2,6 & 54 & 0 & 244 \\
\hline Ouro Preto do Peste & 19,0 & 6,4 & 14,1 & 5,5 & 2,1 & 3,6 & 96 & 7 & 324 \\
\hline Parecis & 13,1 & 0,0 & 6,9 & 8,4 & 0,0 & 2,5 & 387 & 6 & 1863 \\
\hline Pimenta Bueno & 16,3 & 3,8 & 11,3 & 4,8 & 1,1 & 3,0 & 451 & 71 & 2832 \\
\hline Pimenteiras do Oeste & 39,2 & 0,0 & 8,1 & 7,5 & 0,0 & 1,6 & 375 & 49 & 2302 \\
\hline Porto Velho & 17,4 & 10,3 & 13,6 & 8,3 & 2,9 & 5,8 & 3003 & 441 & 19649 \\
\hline Presidente Médici & 13,5 & 7,0 & 9,5 & 4,7 & 1,1 & 2,9 & 60 & 3 & 370 \\
\hline Primavera de Rondônia & 18,6 & 0,0 & 8,0 & 9,2 & 0,0 & 2,6 & 18 & 0 & 113 \\
\hline Rio Crespo & 15,6 & 0,0 & 10,3 & 4,9 & 0,0 & 1,5 & 228 & 23 & 1218 \\
\hline Rolim de Moura & 19,0 & 1,4 & 13,3 & 7,1 & 0,0 & 3,1 & 72 & 4 & 342 \\
\hline Santa Luzia D'Oeste & 14,5 & 3,7 & 8,5 & 5,3 & 0,0 & 2,4 & 42 & 0 & 215 \\
\hline São Felipe D’Oeste & 15,3 & 1,8 & 6,1 & 3,3 & 0,0 & 1,1 & 14 & 0 & 61 \\
\hline São Francisco do Guaporé & 23,2 & 5,1 & 12,1 & 11,1 & 0,0 & 5,5 & 845 & 96 & 5681 \\
\hline São Miguel do Guaporé & 18,1 & 5,4 & 12,0 & 5,4 & 0,0 & 2,1 & 562 & 95 & 3190 \\
\hline Seringueiras & 20,9 & 3,4 & 12,0 & 7,0 & 0,0 & 2,2 & 412 & 43 & 1950 \\
\hline Teixeirópolis & 14,4 & 0,0 & 6,7 & 12,6 & 0,0 & 3,7 & 28 & 0 & 84 \\
\hline Theobroma & 13,3 & 0,0 & 5,7 & 6,0 & 0,0 & 2,0 & 260 & 22 & 1279 \\
\hline Urupá & 17,0 & 2,2 & 9,0 & 4,4 & 0,0 & 1,8 & 93 & 4 & 517 \\
\hline Vale do Anari & 14,8 & 0,0 & 7,6 & 5,9 & 0,0 & 1,5 & 448 & 39 & 2135 \\
\hline Vale do Paraíso & 20,2 & 5,1 & 11,8 & 13,2 & 0,0 & 3,9 & 119 & 2 & 280 \\
\hline Vilhena & 14,7 & 7,5 & 11,8 & 9,1 & 1,5 & 4,1 & 756 & 82 & 3758 \\
\hline
\end{tabular}



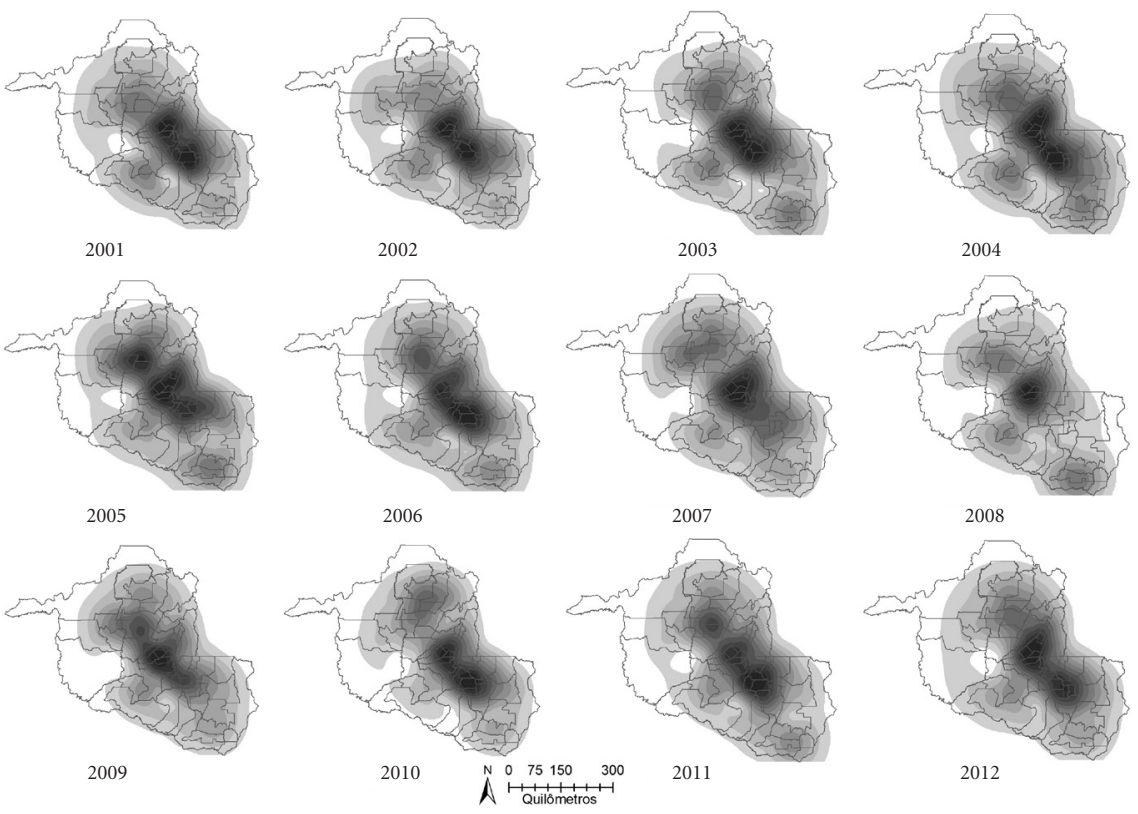

2009

Quilômetros

Figura 2. Distribuição espacial e temporal das taxas padronizadas de mortalidade por doenças cardiovasculares em idosos acima de 60 anos de idade no Estado de Rondônia, no período de 2001 a 2012.

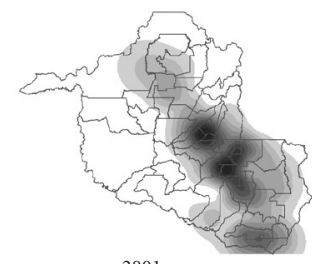

2001

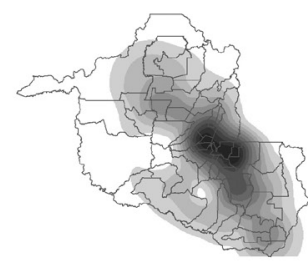

2005

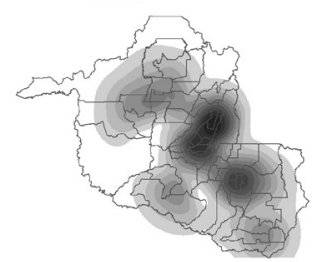

2009

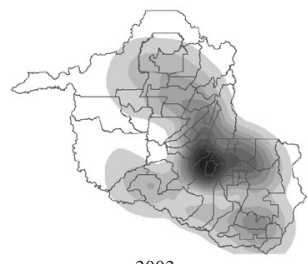

2002

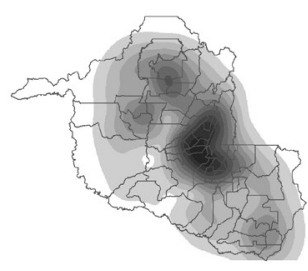

2006

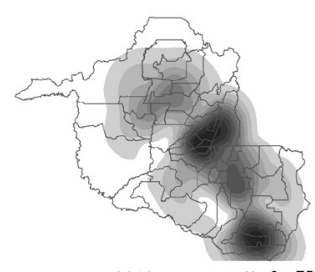

2010

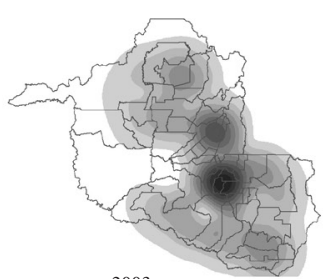

2003
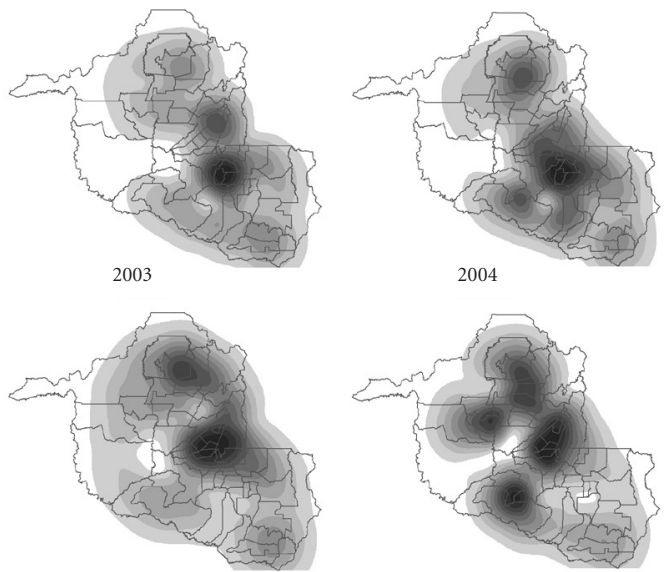

2007

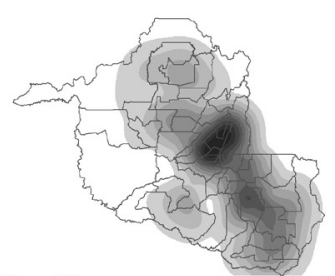

$\stackrel{N}{0} \stackrel{0}{0} 75150 \quad 300$

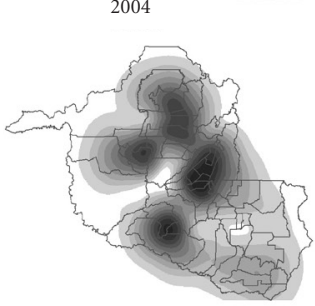

2008

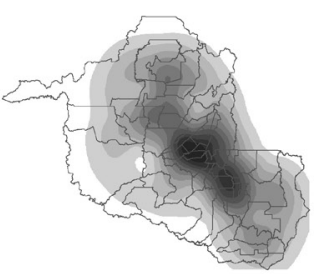

2012

Figura 3. Distribuição espacial e temporal das taxas padronizadas de mortalidade por doenças respiratórias em idosos acima de 60 anos de idade no Estado de Rondônia, no período de 2001 a 2012. 


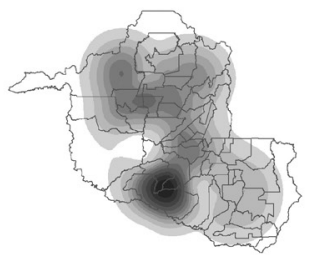

2001

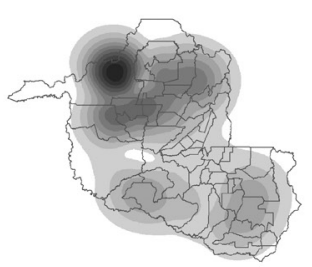

2005

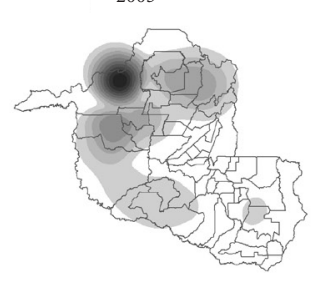

2009

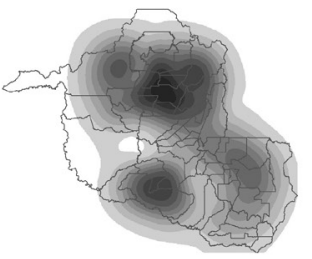

2002
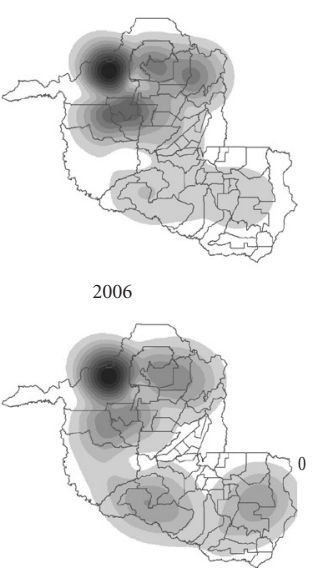

2010

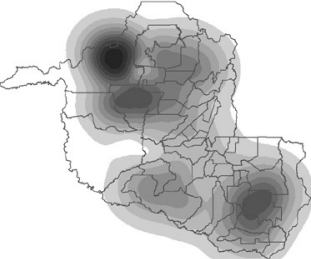

2003

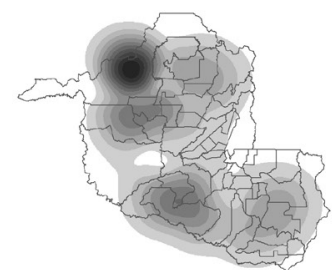

2007

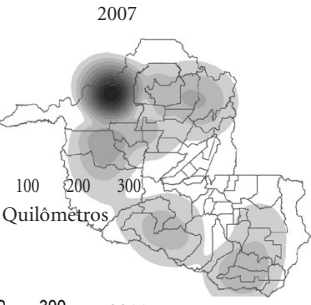

2011

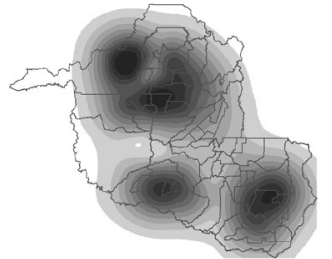

2004
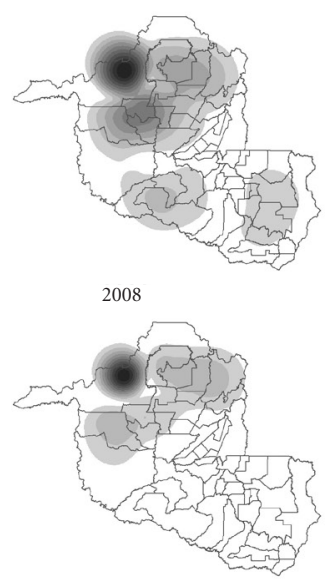

2012

Figura 4. Distribuição espacial e temporal dos focos de queimadas no Estado de Rondônia, no período de 2001 a 2012.

do de 2001 a 2012, não se mostraram diretamente associadas. Entretanto, é notável a grande variabilidade da ocorrência dos focos de queimadas no estado, os quais foram observados em todos os municípios de Rondônia, com maior e menor frequência ao longo da série temporal avaliada.

Estudos anteriores indicaram a tendência de crescimento do número anual de focos para a região, no período de 1998 a $2005^{17}$, assim como foi observada uma redução desse número total, no período de 2005 a 2009, com significativo aumento em $2010^{12}$. As queimadas em Rondônia estão fortemente associadas à abertura e manutenção de áreas destinadas à agropecuária, onde florestas são convertidas em áreas de diferentes monoculturas e também para pastagens, para abrigar um crescente rebanho bovino estimado em cerca de 12 milhões de cabeças, ocupando aproximadamente 6 milhões de hectares plantados com gramínea ${ }^{18}$.

Os municípios da porção norte do estado foram os que mais apresentaram a incidência de "áreas quentes" de queimadas, mas diversos outros municípios registraram relevantes quantidades de focos anuais, como Costa Marques,
São Francisco do Guaporé (ambos no sudoeste) e Vilhena (sudeste). A dispersão dos poluentes relacionados às queimadas podem influenciar a saúde de idosos, visto que os gases e aerossóis emitidos pela queima de biomassa podem ser transportados por milhares de quilômetros das áreas de origem, de acordo com o padrão da circulação atmosférica predominante, pela atuação de movimentos convectivos e de deslocamento das massas de $\operatorname{ar}^{19}$. Desta forma, a exposição aos poluentes originados nas queimadas pode não necessariamente coincidir com o local de ocorrência da queima, razão pela qual os focos de calor devem ser utilizados como indicadores indiretos de exposição.

A diferença da configuração espacial das “áreas quentes" entre as variáveis de análise também foi observada por Rodrigues et al. ${ }^{20}$, em um estudo similar no estado de Rondônia, com o objetivo de descrever a distribuição espaço-temporal dos focos de queimadas e das internações hospitalares por doenças respiratórias em crianças menores de cinco anos de idade, no período de 2001 a 2010. Este estudo também utilizou a metodologia das estimativas de densidade de Kernel, mas 
sua base geográfica de análise foi trabalhada em microrregiões. O refinamento do presente estudo em uma base municipal possibilitou investigar novos parâmetros da análise.

Nunes et al. ${ }^{21}$ buscaram analisar a associação da exposição ao material particulado fino $\left(\mathrm{PM}_{2.5}\right)$ com as taxas de mortalidade por doenças circulatórias em idosos na Amazônia brasileira, no ano de 2005, em 107 microrregiões, incluindo Rondônia. Foi observada uma significativa associação entre as taxas de mortalidade por doenças cardiovasculares e infarto agudo do miocárdio com a porcentagem anual de horas de exposição por $\mathrm{PM}_{2.5}$. Os autores destacaram que as doenças circulatórias, em idosos residentes na Amazônia, têm sido influenciadas pela poluição gerada nas queimadas, especialmente durante a estação seca.

Nas queimadas são emitidos vários poluentes, entre eles monóxido e dióxido de carbono, óxido nitroso e material particulado, além de substâncias altamente tóxicas, quando da exposição aguda ao evento. Idosos com história prévia de doenças cardiorrespiratórias são mais vulneráveis aos efeitos da queima de biomassa ${ }^{9}$. Em geral, os efeitos à saúde humana são o somatório de exposições a diversos agentes, nem sempre fáceis de serem quantificados em estudos ecológicos. Embora os efeitos não estejam bem definidos nos grupos expostos, a poluição pela queima de biomassa é um importante fator de risco para estes grupos vulneráveis.

O uso de satélites é o melhor recurso disponível para estimar a quantidade de queimadas sobre determinada região e tem sido utilizado como indicador indireto das concentrações de poluentes atmosféricos. As limitações envolvem, principalmente, as características de resolução espacial e temporal dos sensores, que podem superestimar ou subestimar a quantidade de focos ${ }^{14}$.

A identificação de áreas críticas quanto à ocorrência de queimadas é um importante instrumento diretivo para a construção de indicadores de saúde sobre os impactos diretos e indiretos à saúde humana. A descrição da situação de saúde de idosos, mesmo que avaliada indiretamente através dos dados de mortalidade, é importante, pois pode propiciar a geração de hipóteses causais além de contribuir para elaboração de programas e políticas de saúde, especificamente neste estudo, para essa população. Considera-se de grande relevância a implementação da vigilância em saúde ambiental com base em indicadores de mortalidade e de morbidade, acompanhado do monitoramento da qualidade do ar nas regiões de queimadas, com foco aos serviços e políticas integradas de saúde e meio ambiente da região Amazônica.

\section{Colaboradores}

Os autores do manuscrito apresentaram as seguintes contribuições: VS Andrade Filho trabalhou na concepção, tabulação, análise, interpretação de dados e redação; PE Artaxo Netto contribuiu na concepção e na revisão crítica do conteúdo; SS Hacon na concepção, interpretação de dados e revisão crítica do conteúdo; CN Carmo trabalhou na análise, interpretação de dados e revisão crítica do conteúdo.

\section{Agradecimentos}

À Júlia Peixoto, pelo importante apoio na elaboração dos mapas de análise.

Este trabalho é uma contribuição do Brazilian Network on Global Climate Change Research, financiado pelo CNPq, FINEP e pelo projeto INOVA ENSP 2015. 


\section{Referências}

1. World Health Organization (WHO). Exposure air to pollution: a major public health concern. Geneva: WHO; 2010.

2. World Health Organization (WHO). Global health risks: Mortality and burden of diseases attributable to selected major risks. Geneva: WHO; 2009.

3. Johnston FH, Henderson SB, Chen Y, Randerson JT, Marlier M, Defries RS, Kinney P, Bowman DMJS, Brauer M. Estimated Global Mortality Attributable to Smoke from Landscape Fires. Environmental Health Perspectives 2012; 120(5):695-701.

4. World Meteorological Organization (WMO). Impacts of Megacities on Air Pollution and Climate. Geneva: WMO; 2012.

5. Arbex MA, Cançado JED, Pereira LAA, Braga ALF, Saldiva PHN. Queima de biomassa e efeitos sobre a saúde. I Bras Pneumol 2004; 30(2):158-175.

6. Arbex MA, Bohn GM, Saldiva PH, Conceição G. Assessment of the effects of sugar cane plantation burning on daily counts of inhalation therapy. J Air Waste Manag Assoc 2000; 50(10):1745-1749.

7. Ignotti E, Valente JG, Longo KM, Freitas SR, Hacon SS, Artaxo P. Impact on human health of particulate matter emitted from burnings in the Brazilian Amazon region. Rev Saude Publica 2010; 44(1):121-130.

8. Gonçalves KS, Castro HA, Hacon SS. As queimadas na região Amazônica e o adoecimento respiratório. Cien Saude Colet 2012; 17(6):1523-1532.

9. Ribeiro H, Assunção JV. Efeitos das queimadas na saúde humana. Estudos Avançados 2002; 16(44):125-148.

10. Artaxo P, Oliveira PH, Lara LL, Pauliquevis TM, Rizzo LV, Pires JRC. Efeitos climáticos de partículas de aerossóis biogênicos e emitidos em queimadas na Amazônia. Rev Brasileira de Meteorologia 2006; 21(3):1-22.

11. Carmo CN, Hacon SS, Longo KM, Freitas S, Ignotti E, Artaxo P. Associação entre material particulado de queimadas e doenças respiratórias na região sul da Amazônia brasileira. Rev Panam Salud Publica 2010; 27(1):10-16.

12. Cardozo FS, Pereira G, Shimabukuro YE, Moraes EC. Avaliação das áreas queimadas no estado de Rondônia, Amazônia Brasileira. Revista Brasileira de Cartografia 2014; 66(3):705-716.

13. Fearniside PM. Desmatamento na Amazônia: dinâmica, impactos e controle. Acta Amazônica 2006; 36(3):365-400
14. Instituto Nacional de Pesquisas Espaciais (INPE). SIG Queimadas: monitoramento de focos. [acessado 2015 jan 31]. Disponível em: http://www.dpi.inpe.br/proarco/ bdqueimadas/

15. DATASUS. Sistema de Informações sobre Mortalidade SIM. [acessado $2014 \mathrm{dez} 1$ 1]. Disponível em: http://tabnet.datasus.gov.br/cgi/sim/dados/indice.htm

16. Instituto Brasileiro de Geografia e Estatística (IBGE). [acessado 2015 jan 31]. Disponível em: http://www. ibge.gov.br/home/

17. Casto HA, Gonçalves KS, Hacon SS. Tendência da mortalidade por doenças respiratórias em idosos e as queimadas no estado de Rondônia/Brasil - período entre 1998 e 2005. Ciência e Saúde Coletiva 2009; 14(6):20832090.

18. Rondônia. Secretaria de Estado do Desenvolvimento Ambiental (SEDAM). Plano de prevenção, controle e alternativas sustentáveis ao desmatamento em Rondônia. 2009 - 2015. Porto Velho: SEDAM; 2009.

19. Freitas SR, Longo KM, Silva Dias MAF, Silva Dias PL, Chatfield R, Prins E, Artaxo P, Recuero F. Monitoring the transport of biomass burning emissions in South America. Environmental Fluid Mechanics 2005; 5(1):135-167.

20. Rodrigues PCO, Ignotti E, Hacon SS. Distribuição espaço-temporal das queimadas e internações por doenças respiratórias em menores de cinco anos de idade em Rondônia, 2001 a 2010. Epidemiol. Serv. Saúde 2013; 22(3):455-464.

21. Nunes KVR, Ignotti E, Hacon SS. Circulatory disease mortality rates in the elderly and exposure to $\mathrm{PM}_{25}$ generated by biomass burning in the Brazilian Amazon in 2005. Cad Saude Publica 2013; 29(3):589-598.

Artigo apresentado em $01 / 06 / 1015$

Aprovado em 06/10/2015

Versão final apresentada em 08/10/2015 